\title{
Insight into RIPK4 mutations I121N and T184I involved in popliteal pterygium syndrome lethal type using computational methods
}

Mirza Jawad ul Hasnain ${ }^{1}$, Amina Sarwar ${ }^{1}$, Tehreem Anwar ${ }^{1}$, Tahera Aslam $^{1}$ and Muhammad Tariq Pervez ${ }^{*}$

1. Department of Bioinformatics and Computational Biology, Virtual University of Pakistan, Lahore, Pakistan

*Corresponding author's email: m.tariq@vu.edu.pk

\section{Citation}

Mirza Jawad ul Hasnain, Amina Sarwar, Tehreem Anwar, Tahera Aslam and Muhammad Tariq Pervez. Insight into RIPK4 mutations I121N and T184I involved in popliteal pterygium syndrome lethal type using computational methods. Pure and Applied Biology. Vol. 11, Issue 2, pp670-683. http://dx.doi.org/10.19045/bspab.2022.110068

\begin{tabular}{llll}
\hline \hline Received: 21/06/2021 & Revised: 26/08/2021 & Accepted: 22/09/2021 & Online First: 26/09/2021
\end{tabular}

\section{Abstract}

In this study, computational analysis has been conducted to analyze the wild and mutant RIPK4 (ReceptorInteracting Serine/Threonine-Protein Kinase 4) gene using in silico approach. RIPK4 is a receptorinteracting serine/threonine-protein kinase that gives a large impulse for the development of stratified epithelium in the same manner as an explicit transcriptional recipient for TP63 that is responsible for the regulation of molecular events involved in the prevention of Pterygium syndrome. Homozygous transitional mutations in RIPK4, Ile121Asn, and Thr184Ile are reported to be the ground reason of functional impairment of RIPK4 cause Lethal Type of Popliteal Pterygium Syndrome. Several in silico approaches were performed through different bioinformatics software and online tools for Structure prediction, Evolutionary analysis, Pocket identification, and docking analysis. Results of protein superimposition clearly showed the change in the structures of wild type and mutant type of RIPK4, where both mutations alter the normal structure of protein leading to change in its interaction site and conformations due to which the interacting capabilities of RIPK4 are affected that might be resulting in the alteration of function. Evolutionary analysis of RIPK4 indicated a close phylogenetic association between Homo sapiens and Pan troglodytes among 15 orthologs. In silico analysis and computational methods have opened many doors for understanding several diseases in a time and cost-effective way. Protein-ligands docking effectively proved the structural alteration caused by the said mutations and can be used in developing drug therapies, accurate diagnosis, and better therapeutic strategies for the patients.

Keywords: Docking; Mutation; Pterygium syndrome; Phylogenetic analysis; RIPK4; Superimposition

\section{Introduction}

Popliteal Pterygium syndrome is a condition in which the development of genitals, face, and skin is affected [1]. Many disorders like birth with a cleft lip, cleft palate, pits near the center of the lower lip have been reported in this syndrome [2]. Popliteal Pterygium syndromic infants may be born with webs of skin on the backs of the legs across the knee joint which can impair mobility. The autosomal-recessive form of this syndrome is a rare but frequently lethal disorder characterized by marked popliteal Pterygium associated with multiple congenital malformations. Almost 25,100 cases with 
Popliteal Pterygium syndrome have been reported to date world widely[3].

Receptor-Interacting Serine/ThreonineProtein Kinase 4 (RIPK4) is involved in causing Popliteal Pterygium Syndrome Lethal Type [4, 5]. RIPK4-receptor consisting of 8 exons, located at 21q.22.3 cytogenetic position with a size of $27.7 \mathrm{~kb}$ [6]. This receptor is widely expressed in embryonic and mature tissues, such as myocardium liver, skeletal muscles, and lungs, as well as involved in a variety of signaling pathways to play a vital role in regulating cell apoptosis, proliferation, differentiation, and inflammatory response [7].

It consists of an N-terminal kinase domain and a C-terminal region that consists of 11 ankyrin repeats [8]. RIPK4 is also essential for the differentiation of keratinocytes and epithelial cells, skin inflammation, and healing of wound surface, as well as for tumorgenesis.

Against its different roles and function, it possesses several alternative aliases like $A N K K 2, D I K, P K C$ - delta- interacting protein kinase, PKK-protein kinase, C-associated kinase, and RIPK4. As RIPK4 shows an interaction with the protein kinase $\mathrm{C}$-delta $(\mathrm{PKC}-\delta)$, named as $\mathrm{PKC}-\delta$-interacting protein kinase [6]. Its associated pathways include Wnt/ Hedgehog/ Notch [9] and NFkappa-B signaling pathways[10] and are linked with gene ontology (GO) annotations that indicate transferase activity [11] and protein tyrosine kinase activity [12].

Amongst the functions of RIPK4 in human beings, the most significant role is in the development of stratified epithelium as an explicit transcriptional recipient for TP63 [13, 14]. TP63 is responsible for the regulation of molecular events involved in the prevention of Pterygium Syndrome [15]. The most significant mutations of RIPK4 causing Popliteal Pterygium Syndrome are reported at positions 121 and 184; both cause a loss in functionality of RIPK45-10. A homozygous transversion (c.362T $>\mathrm{A}$ ) mutation, that is caused by the substitution of conserved isoleucine with asparagine, is reported at amino acid position 121 (p.I1e121Asn) in the serine/threonine kinase domain of the protein. Another pathogenic point mutation reported at amino acid position 184 (p.Thr184I1e) is also a homozygous transition (c.551C $>\mathrm{T}$ ) mutation, in which threonine is substituted by isoleucine. Both the I1e121 and Thr184 positions are revealed to be critical for protein stability, kinase, and catalytic activity of RIPK4. RIPK4 mediates activation of the nuclear factor- $\kappa \mathrm{B} \quad(\mathrm{NF}-\kappa \mathrm{B})$ signaling pathway and is required for keratinocyte differentiation and craniofacial and limb development [10].

In the current study, computational analysis has been carried out for normal RIPK4 gene along with a frameshifting, truncating mutation in the gene i.e., p. Ile121Asn and Thr184Ile. To further investigate the functionality of RIPK4 and the effects of mutations at both positions I121A and T184I, Homology Modeling and its Evaluation were performed and scrutinized. Protein-protein and protein-ligand docking of wild structure and mutant structures were performed with associated ligands and neighboring proteins of RIPK4 for studying the structural alterations in structures. Furthermore, Evolutionary analysis was also carried out for a better understanding of the RIPK4 gene among the orthologs of Homo sapiens.

\section{Materials and Methods}

A schematic representation of the methodology used in the current study is given in (Fig. 1). The sequence of RIPK4 was retrieved from OMIM database (Online Mendelian Inheritance in Man) MIM\# 263650, an Online Catalog of Human Gene and Genetic disorders[16]. This retrieved sequence was used for the secondary structure prediction of RIPK4 wild type and 
both mutants I121A and T184I through an online tool PSIPRED [17]. Although the crystal structure of murine receptorinteracting protein kinase 4 (RIPK4) with PDB-ID 5WNI-L for Mus musculus has been reported by X-ray diffraction method, but not covered the entire sequence. Due to the unavailability of full human RIKP4 protein structure, we used MODELLER [18] to predict the 3-D structures of all 3 sequences of RIPK4 (Wild type, I121A, and T184I mutants) using the threading approach. The crystal structure of murine receptorinteracting protein kinase 4 (RIPK4) with PDB-ID 5WNI-L was taken as one of the four templates (5WNI-L, 6ES0, 4C8B, and 5NG0) of MODELLER. FOLDX [19] was used for the energy minimization, structure repairing, and building of the structures of mutants I121N and T184I of RIPK4 protein. All of the three tertiary structures were then evaluated using RAMPAGE and ERRAT server [20].

Superimposition of the wild and mutant structures of RIPK4 using PyMol [21, 22] was performed for 3D protein structure visualization and to identify the effect on structure and behavior caused by mutations. CASTp (Computed Atlas Surface Topography of Proteins)[23] was used to carry out Pocket identification of wild and mutant protein structures. Then identified pockets on the wild-type RIPK4 and its mutants were compared to detect the effect of the mutation on the binding sites of the protein. In docking analysis, the Protein minimization of predicted structures of RIPK4 and its mutants (I121N, and T184I) was performed using 3Drefine[24]. The closely related 50 neighbor proteins of RIPK4 were retrieved from the STRING database using the medium confidence score of 0.4 for all active interaction sources in full STRING network type. The network was further filtered on high confident interactions with a score $=0.7$ for both functional \& physical protein associations, medium confidence score $=0.4$ for experimental interaction source only. Common protein from the network was selected and docked with native and mutant models of RIPK4 protein to see the impact of the mutation on the residual interactions. In protein-protein Docking, interactions of RIPK4 and its I121N and T184I mutants with neighbor protein was performed using the Hex Protein Docking Software [25]. In protein-ligand docking, RIPK4 and its both mutants (I121A and T184I) were docked with ligands (4GD, 4GF, 6GE, 6QY, 6R0, 8BY, 8YB, 9LI, 9YS, 9YY, 33Y, 064) using PyRx software [26] to see that how different a structurally modified protein interacts with surrounding ligands. PyRx is a valuable tool for Computer-Aided Drug Designing that includes a docking wizard with an easy-to-use user interface.

The interacting residues of wild and mutant structures with neighbor protein and ligands were further analyzed using LigPlus. For visualization of protein-protein interaction, the DimPlot was used and LigPlot was used for protein-ligand interactions[27]. The resultant $2 \mathrm{D}$ depictions were compared to identify any possible changes in interacting residues caused by the mutation at positions I120N and T184I [28].

In Evolutionary Analysis, for this study 15orthologs of Homosapien RIPK4 were selected using Ensembl genome browser 95 (https://www.ensembl.org/) on the basis of similarity in their sequences. These considered species include Ailuropoda melanoleuca, Bos taurus, Canis lupus familiaris, Capra aegagrus hircus, Cavia porcellus, Danio rerio, Equus caballus, Felis catus, Gallus domesticus, Macaca mulatta, Marmota marmota, Mus musculus, Ovis aries musimon, Pan troglodytes and Sus scrofa. The sequences of all these orthologs were retrieved for the construction of a phylogenetic tree with Pairwise Sequence Alignment (PSA) and Multiple Sequence 
Alignment (MSA). In PSA, the RIPK4 sequence was kept constant and aligned with 15 orthologs to identify the similarity among RIPK4 and its orthologs using EMBOSS Needle [29, 30]. For MSA an online tool, TCoffee [31] was used providing sequences of orthologs in text format along with the sequence of RIPK4 wild type. The results of $\mathrm{T}$-Coffee provided the aligned sequences with the identification of good, average and bad alignment with the documentation of similarity among orthologs at particular regions. Both points of mutation (I121N and T184I) were taken as a reference point to identify the conservation in orthologs. The amino acids on both points of mutation were observed either to be conserved or nonconserved by using the results obtained from T-Coffee MSA (https://www.ebi.ac.uk/Tools/msa/tcoffee/). The FASTA format sequences of all the RIPK4 orthologs retrieved earlier were aligned in MEGA7 [32] performing MSA using CLUSTALW [33]. The retrieved aligned file was used for phylogenetic tree construction using the Neighbor-Joining method along with the bootstrap method to construct the scored phylogenetic tree. The resultant phylogenetic tree formed using bootstrap [33, 34] values were then interpreted to identify the evolution among orthologs of the RIPK4 gene.

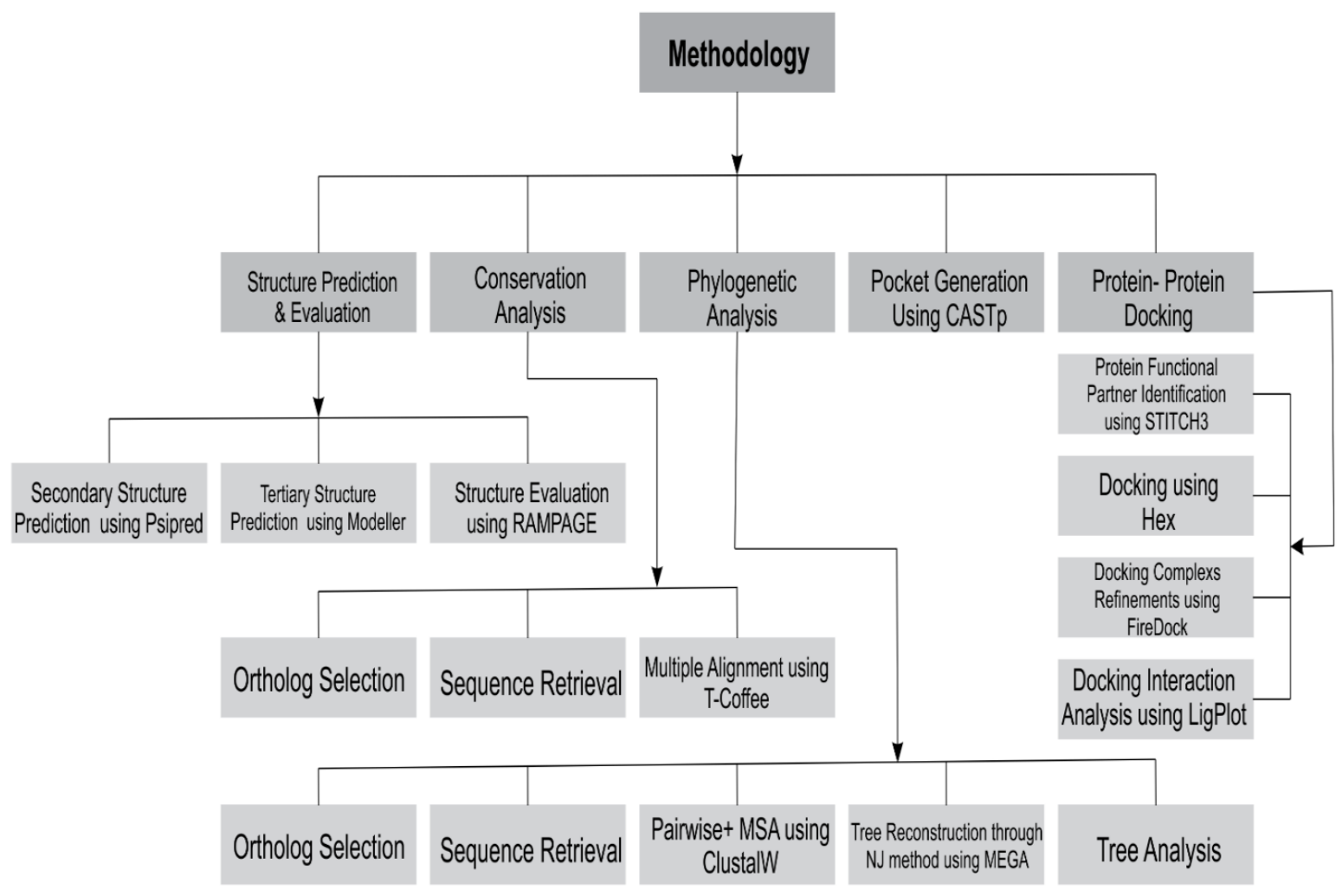

Figure 1. Schematic workflow of processing methodology for RIPK4 gene 


\section{Results}

Structure prediction and evaluation Secondary structure prediction

The secondary structure predicted using PSIPRED showed variation in the number of residues of coils, helix, and sheets among the wild and mutant sequences of RIPK4, which confirms the effect of the mutation on the structure. The change in the side-chain orientation due to mutation at positions 121 and 184 alters the overall structure of both mutants (I121A and T184I). The (Table 1) shows the comparison between the secondary structures of RIPK4 and its both mutants (I121A and T184I).

The number of residues in helices, strands, and coils of the secondary structures of RIPK4 and both I121A and T184I mutants is shown in (Fig. 2).

\section{Tertiary structure prediction}

The coiling and recoiling of the predicted structure resulted in the formation of the tertiary structure of the wild-type, predicted via MODELLER (Fig. 3). Mutant structures of RIPK4 (I121N and T184I) were built using FOLDX. After the energy minimization, the structural variations among wild-type and mutant structures were checked by the superimposition of wild type as a reference with mutants via structural alignment using PyMol. The superimposition shows that the mutation caused a change in structure; (Fig.4) showed the structural comparison of wild type with I121N and T184I mutants with residual change at positions 121 and 184 respectively.

Table 1. Secondary structure comparison among wild type sequence and sequences with the mutation at positions 121 and 184

\begin{tabular}{|c|c|c|c|c|c|c|}
\hline $\begin{array}{c}\text { Protein } \\
\text { Structure }\end{array}$ & $\begin{array}{c}\text { Alpha } \\
\text { Helix }\end{array}$ & Percentage & $\begin{array}{c}\text { Extended } \\
\text { Strand }\end{array}$ & Percentage & $\begin{array}{c}\text { Random } \\
\text { Coil }\end{array}$ & Percentage \\
\hline Wild Type & 285 & $36.35 \%$ & 116 & $14.80 \%$ & 383 & $48.85 \%$ \\
\hline T184I Mutant & 285 & $36.35 \%$ & 118 & $15.05 \%$ & 381 & $48.60 \%$ \\
\hline I121N Mutant & 279 & $35.59 \%$ & 116 & $14.80 \%$ & 389 & $49.60 \%$ \\
\hline
\end{tabular}

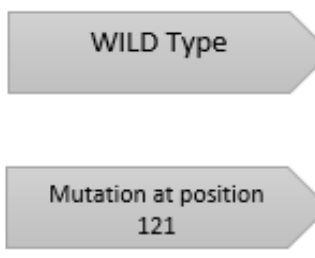

Mutation at position 184
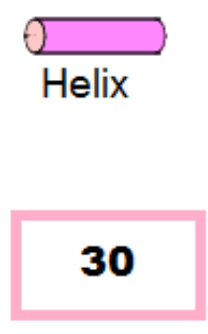

31

32

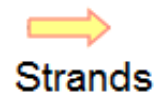

coils

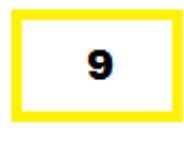

$\mathbf{9}$

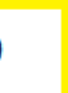

9

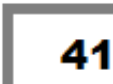

41

42

Figure 2. Descriptive diagram showing the total number of residues in Alpha Helix, Beta Strands, and Coils in all 3 secondary Structures 


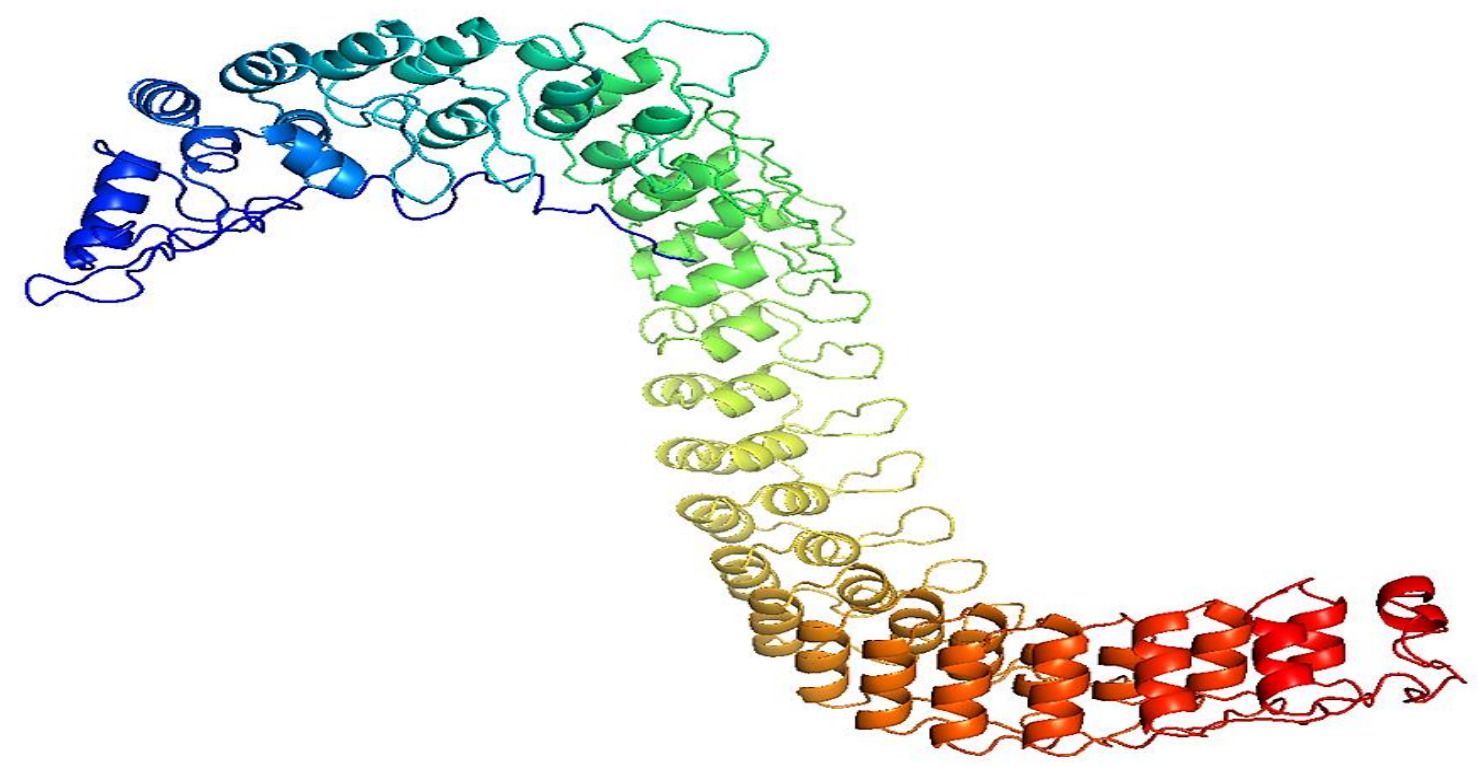

Figure 3. Three-dimensional protein structure of RIPK4 wild type

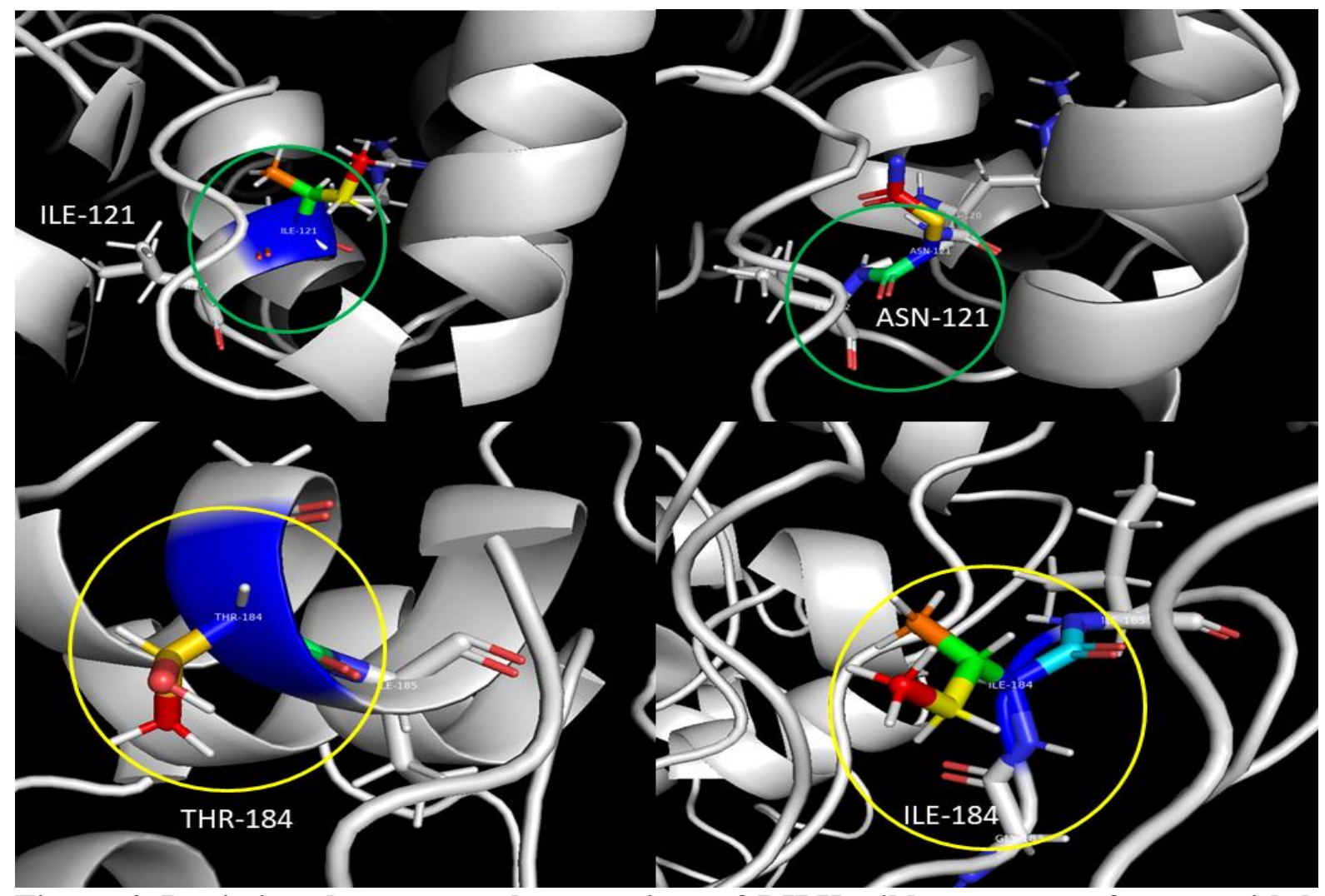

Figure 4. Depicting the structural comparison of RIPK wild type as a reference with both mutants at position 121 and 184 respectively. Green circled is the structural comparison at I121N and yellow circled is the structural comparison at T184I 


\section{Structure evaluation}

The predicted structures were evaluated using the Ramachandran Plot approach of RAMPAGE software to detect the percentage of Residues lying in the favored region to identify the reliability of the predicted structures. The maximum number of residues reclines in the favored region giving the higher reliability of predicted structures.
Even though mutation caused the change in structure formation, it also affects the number of favored regions as well as outliners, leading to a change in the functionality of protein causing several diseases. The ERRAT evaluation provided the quality factor score of all three predicted structures that proved the good quality of structures shown in (Table 2).

Table 2. Structural evaluation of predicted structures. ERRAT quality factor refers to that $\%$ of proteins whose error value falls below $95 \%$ of the rejection limit. RAMPAGE results show which amino acid residues angles lie in which area of the Ramachandran plot

\begin{tabular}{|c|c|c|c|c|}
\hline Sequence Type & Favored Regions & Allowed Regions & Outlier Regions & $\begin{array}{c}\text { ERRAT Quality } \\
\text { Factor }\end{array}$ \\
\hline Wild Type & $\begin{array}{c}601 \text { residues } \\
76.7 \%\end{array}$ & $\begin{array}{c}118 \text { residues } \\
15.1 \%\end{array}$ & $\begin{array}{c}63 \text { residues } \\
8.1 \%\end{array}$ & $86.34 \%$ \\
\hline $\begin{array}{c}\text { Mutation at } \\
\text { position I121N }\end{array}$ & $\begin{array}{c}562 \text { residues } \\
71.9 \%\end{array}$ & $\begin{array}{c}134 \text { residues } \\
17.1 \%\end{array}$ & $\begin{array}{c}86 \text { residues } \\
11 \%\end{array}$ & $82.82 \%$ \\
\hline $\begin{array}{c}\text { Mutation at } \\
\text { position T184I }\end{array}$ & $\begin{array}{c}612 \text { residues } \\
78.3 \%\end{array}$ & $\begin{array}{c}121 \text { residues } \\
15.5 \%\end{array}$ & $\begin{array}{c}49 \text { residues } \\
6.3 \%\end{array}$ & $83.05 \%$ \\
\hline
\end{tabular}

\section{Structure refinement}

All three structures of RIPK4 (Wild type and its both mutants I121 A and T184I) predicted using MODELLER were refined using the $3 \mathrm{D}^{\text {refine }}$ tool to minimize the energy of the system. The minimized structures were further used for docking analysis rather than the actual predicted structures.

\section{Pocket generation}

Before docking analysis, active sites of the RIPK4 protein and its mutants were identified using CASTp, an online tool for the identification of active sites. The total number of active sites identified in wild type structure of RIPK4 was 130 out of which one pocket contained amino acid Ile at position 121 but no active site contained amino acid Thr at position 184 . The number of pockets in the I121A was minimized to 119 including 3 pockets that contained Thr at position 184 . The T184I mutant contained a total of 112 pockets out of which 3 pockets contained Ile at position 184. This showed that the change in structures lead to the change in the functionality of protein due to mutation.
Mutation at position 121 of RIPK4 (I121A) not only affected active sites in a way that it ended up reducing the number of pockets but also it made the non-active part of the protein (position 184 containing Thr) functional while the number of pockets containing position 121 remained the same showing no specific change. Mutation at position 184 of RIPK4 (T184I) also reduced the number of active sites while 3 pockets were identified containing position 184 which means that the mutation caused the active sites to become non-functional. Besides that, it also made non-active amino acid at position 184 become functional as well as the active amino acid at position 121 become inactive as no pocket contained position 121.

\section{Docking analysis}

\section{Protein-ligand docking}

The ligands retrieved from RCSB Protein Data Bank were 4GD, 4GF, 6GE, 6QY, 6R0, 8BY, 8YB, 9L1, 9YS, 9YY, 33Y, and 064. All these ligands were docked with wild type and both mutant type protein structures using PyRx because upon docking they gave 
binding affinity less than -6 and showed great variation in values among wild type docked compounds in comparison with both mutants. The interacting residues vary in the wild-type and both mutants docked compounds. The difference in the binding affinity of interacting residues can be seen in (Table 3).

Table 3. Binding affinities of ligands bound with wild type and mutant structures in Kcal/mol

\begin{tabular}{|c|c|c|c|}
\hline \multirow{2}{*}{ Ligand } & \multicolumn{3}{|c|}{ Binding Affinity (Kcal/mol) } \\
\cline { 2 - 4 } & Docked with Wild structure & Docked with mutant at 121 & Docked with mutant at 184 \\
\hline 4GD & -6.8 & -6.6 & -8.9 \\
\hline 4GF & -7.7 & -8.0 & -8.6 \\
\hline 6GE & -6.3 & -7.8 & -8.9 \\
\hline 6QY & -6.8 & -6.4 & -8.5 \\
\hline 6R0 & -8.1 & -8.6 & -9.8 \\
\hline 8BY & -7.2 & -7.5 & -6.6 \\
\hline 8YB & -8.0 & -8.2 & -7.8 \\
\hline 9L1 & -7.5 & -8.6 & -8.7 \\
\hline 9YS & -6.0 & -6.9 & -7.1 \\
\hline 9 YY & -7.2 & -8.0 & -8.7 \\
\hline 33Y & -7.2 & -7.8 & -8.4 \\
\hline 064 & -6.8 & -8.3 & -7.6 \\
\hline
\end{tabular}

\section{Protein-protein docking}

To retrieve neighbor proteins of RIPK4 with maximum association score, we used the STRING database and found 10 (BTRC, FICD, GRHL3, IRF6, KDF1, LIMS2, PRKCB, PRKCD, PTCHD1, VEGFA) closely related proteins from the network (Fig. 5A). Among these 10 neighbor proteins, only three proteins (IRF6, PRKCD, LIMS2) were found to have a confidence score greater than 0.7 (Fig. 5B). PRKCD was the most related protein among all of these. The most closely related neighbor protein PRKCD was docked with wild type and its I121A and T184I mutants in ascending order of score using Hex. The correlation type was set to Shape + Electro + DARS and all the other parameters were set to default. The interaction of PRKCD with RIPK4 and its I121A and T184I mutants was found adequate upon evaluation. All 3 docked compounds were then analyzed using DimPlot of LigPlus to identify the difference among interacting residues shown in (Fig. 6). All docked structures contained chains A and $\mathrm{B}$, but the interacting residues and their corresponding hydrophobic and hydrophilic interactions differ. The interacting residues that differ can be observed in (Table 4).

\section{Evolutionary analysis}

Fifteen orthologs of RIPK4 were selected and retrieved from the Ensemble database. Pairwise alignment of Homo sapiens with selected orthologs was performed using 
EMBOSS platform with EMBOSS Needle to identify the score of evaluation among all. Among all the orthologs, Macaca mulatta was identified to be the most similar (98.2\%), carrying maximum identity $(99.0 \%)$ with a score of 4010.5 while Danio rerio was detected as the least related with human RIPK4 with a similarity of $56.7 \%$ and identity of $69.6 \%$ with the highest number of gaps $(11.1 \%)$ having the least score of 2409.0.

\section{Phylogenetic analysis}

Multiple sequence alignment was performed using T-coffee to identify the conservation of Isoleucine (Ile) at position 121 and Threonine
(Thr) at position 184 among all orthologs shown in (Fig. 7). Both positions in RIPK4 protein were identified to be fully conserved using $100 \%$ conservation among all orthologs. The maximum conservation indicates the importance of both positions functionally. The phylogenetic tree using all 15 orthologs was constructed using MEGA7 to observe the evolution. The bootstrap method was performed to identify and verify the most closet ancestors shown in (Fig. 8). The nearest ancestor of humans was found to be Pan troglodytes with almost 99 support values.

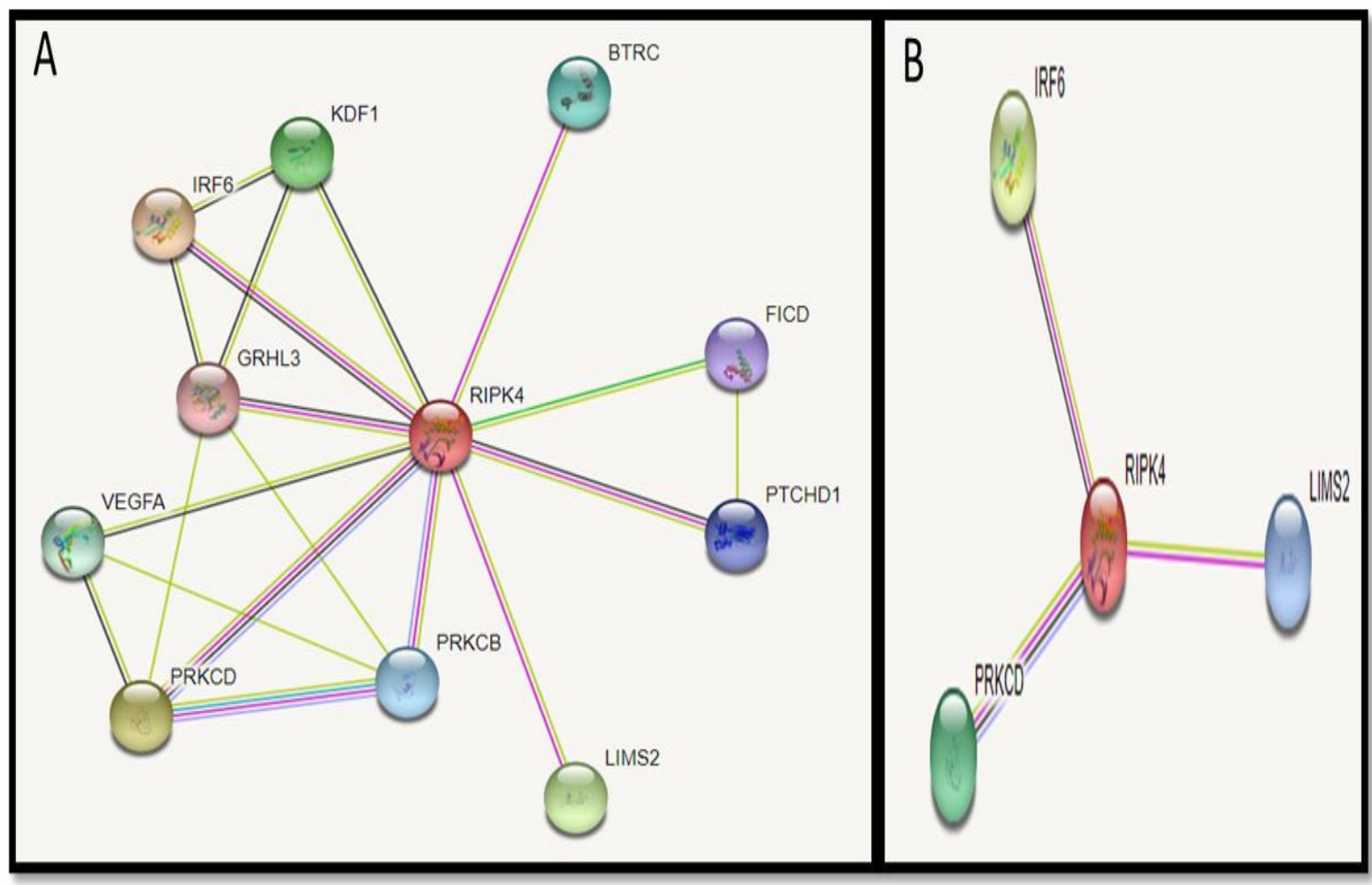

Figure 5. Protein-Protein network from STRING database: A) network of RIPK\$ associated proteins with high confident interactions with score $=0.7$ for both functional \& physical protein associations, B) network of RIPK\$ associated proteins with medium confidence score $=0.4$ for experimental interaction source only 


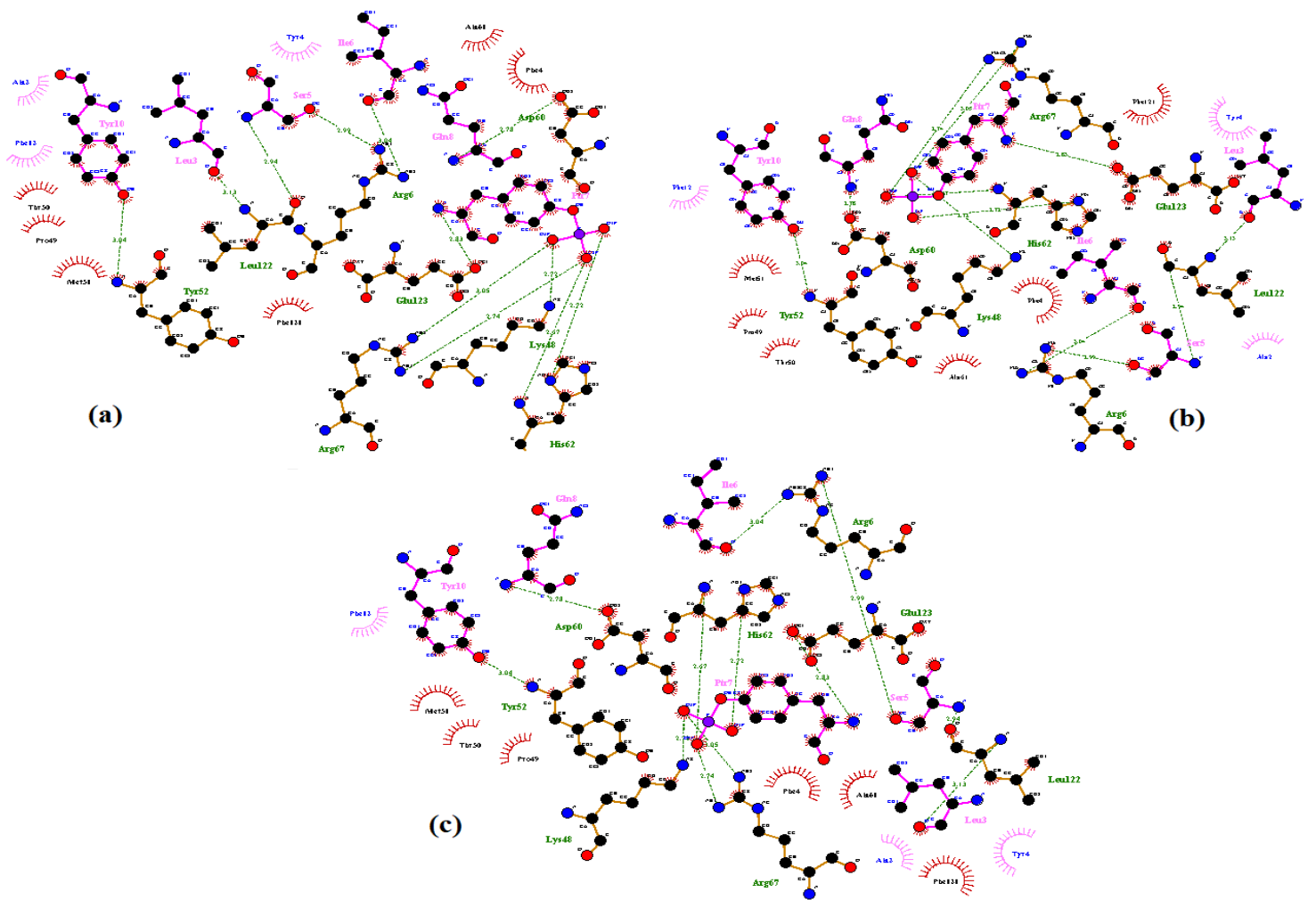

Figure 6. Ligplot of interacting residues. (a) Docking interaction analysis results of wild-type structure of RIPK4 with PKRCD protein (b) Docking interaction analysis results of the mutant structure of RIPK4 carrying mutation at position 121 with PKRCD protein (c) Docking interaction analysis results of the mutant structure of RIPK4 carrying mutation at position 184 with PKRCD Protein.

Table 4. Wild type RIPK4 and its mutants showing the interacting residues and their hydrophobic and hydrophilic interactions that differ in docking with PKRCD protein

\begin{tabular}{|c|c|c|c|c|}
\hline \multirow{2}{*}{ Receptor - Ligand } & \multicolumn{2}{|c|}{ Hydrogen Bond Interaction } & \multicolumn{2}{|c|}{ Hydrophobic Interactions } \\
\hline & Receptor & Ligand & Receptor & Ligand \\
\hline $\begin{array}{c}\text { Wild type } R I P K 4- \\
\text { PKRCD }\end{array}$ & $\begin{array}{c}\text { Tyr52, Leu22, } \\
\text { Arg6, Glu123, } \\
\text { Lys48, Arg67, His } \\
\text { 62, Asp60 }\end{array}$ & $\begin{array}{l}\text { Gln8, Ptr7, Ile6, } \\
\text { Ser5, Leu3 }\end{array}$ & $\begin{array}{l}\text { Thr50, Pro49, } \\
\text { Met51, Phe121, } \\
\text { Phe4, Ala61 }\end{array}$ & $\begin{array}{c}\text { Phe12, Ala2, } \\
\text { Tyr4 }\end{array}$ \\
\hline $\begin{array}{l}\text { Mutant } 121- \\
\text { PKRCD }\end{array}$ & $\begin{array}{c}\text { Tyr52, Asp60, } \\
\text { Lys48, His62, } \\
\text { Arg67, Glu 123, } \\
\text { Arg6, leu22 }\end{array}$ & $\begin{array}{l}\text { Tyr10, Gln8, Ptr7, } \\
\text { Ile6, Ser5, leu3 }\end{array}$ & $\begin{array}{l}\text { Thr50, Pro49, } \\
\text { Met51, Ala61, } \\
\text { Phe4, Phe121 }\end{array}$ & $\begin{array}{c}\text { Phe12, Tyr4, } \\
\text { Ala2 }\end{array}$ \\
\hline $\begin{array}{l}\text { Mutant } 184- \\
\text { PKRCD }\end{array}$ & $\begin{array}{l}\text { Tyr52, Asp60, } \\
\text { Lys48, Arg67, } \\
\text { His62, Arg6, } \\
\text { Glu123, Leu122 }\end{array}$ & $\begin{array}{l}\text { Gln8, Tyr 10, Ile6, } \\
\text { Ptr7, Ser5, Leu3 }\end{array}$ & $\begin{array}{l}\text { Met51, Thr50, } \\
\text { Pro 49, Phe4, } \\
\text { Ala61, Phe121 }\end{array}$ & $\begin{array}{c}\text { Phe12, Ala2, } \\
\text { Tyr4 }\end{array}$ \\
\hline
\end{tabular}




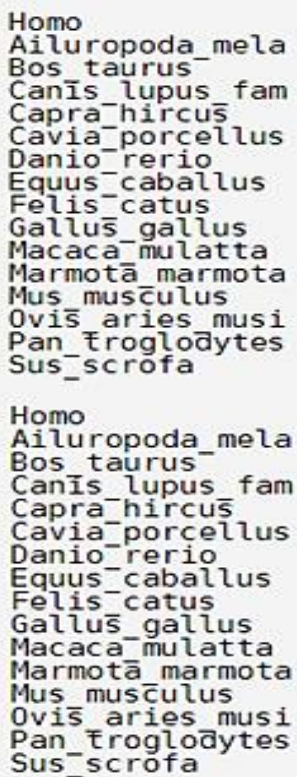

HETAVGMNFLHCMAPPLLHLDLKPANILLDAHYHVKISDFGLAKCNGLS HETAVGMNFLHCMAPPLLHLDLKPANILLDAHYHVKISDFGLAKCNGLS HETAVGMNFLHCMAPPLLHLDLKPANILLDAHYHVKISDFGLAKCNGLS HETAVGMNFLHCMAPPLLHLDLKPAN ILLDAHYHVK ISDFGLAKCNGLS HETAVGMNFLHCMAPPLLHLDLKPANILLDAHYHVKISDFGLAKCNGLS HETAVGMNFLHCMSPPLLHLDLKPANILLDAHYHVKISDFGLAKCTGLS HETAVGMNFLHCMNPPLLHLDLKPANILLDAHYHIKISDFGLARWNGFA HETAVGMNF HCMSPPL H DIKPANI D AHYHVKISDFGLAKCNGL T TVGMN 作 作

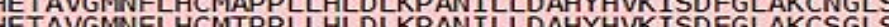
年 年 年 HETAVGM FLHCCAPPLLHLDLKPANILLDAHYHVKISDFGLAKCNGLS HETAVGMNFLHCMAPPLLHLDLKPANILLDAHYHVKISDFGLAKCNGLS ASEPLPWDLRF ASEPLPWDLRF AYLPPERIREKSRLFDTKHDVYSFAIVIWGVLTOKKPFADEKNILHIMV AYLPPERIREKSRLFDTKHDVYSFAIVIWGVLTOKKPFADEKNILHIMV AYLPPERIREKSRLFDTKHDVYSFAIVIWGVLTOKKPFADEKNILHIMV AYLPPERIREKSRLFDTKHDVYSFAIVIWGVLTOKKPFADEKNILHIMV AYLPPERIREKSRLFDIKHDVYSFAIVIWGVLTOKKPFADEKNILHIMV AYLPPERIREKSRLFDTKHDVYSFAI VLWGVLTOKKPFADEKNILHIMV AYLPPERIIEKDRVSDTKHDVYSFSIVIWGILTOKKPYOGENNILHIMV AYLPPERIREKSRLFDTKHDVYSFAI VMWGVLTOKKPFADEKNILHIMV AYLPPERIREKSRLFDTKHDVYSFAIVIWGVLTOKKPFADEKNILRIMV AYLPPERIKEKNRCFDTKHDVYSFSI VIWGVLTOKKPFAEENNILHIMV AYLPPERIREKSRLFDTKHDVYSFAIVIWGVLTOKKPFADEKNILHIMV AYLPPERIREKSRLFDTKHDVYSFAIVIWGVLTOKKPFADEKNILHIMV AYLPPERIREKSRLFDTKHDVYSFAIVIWGVLTOKKPFADEKNILHIMM AYLPPERIREKSRLFDTKHDVYSFAIVIWGVLTOKKPFADEKNILHIMV AYLPPERIREKSRLFDTKHDVYSFAIVIWGVLTOKKPFA .......... AYLPPERIREKSRLFDTKHDVYSFAI VLWGVLTQKKPFADEKNILHVMV

Figure 7. MSA results retrieved using T-coffee showing only the part of sequence carrying (a) position 121, indicating the conserved Ile. (b) position 184, indicating the conserved Thr

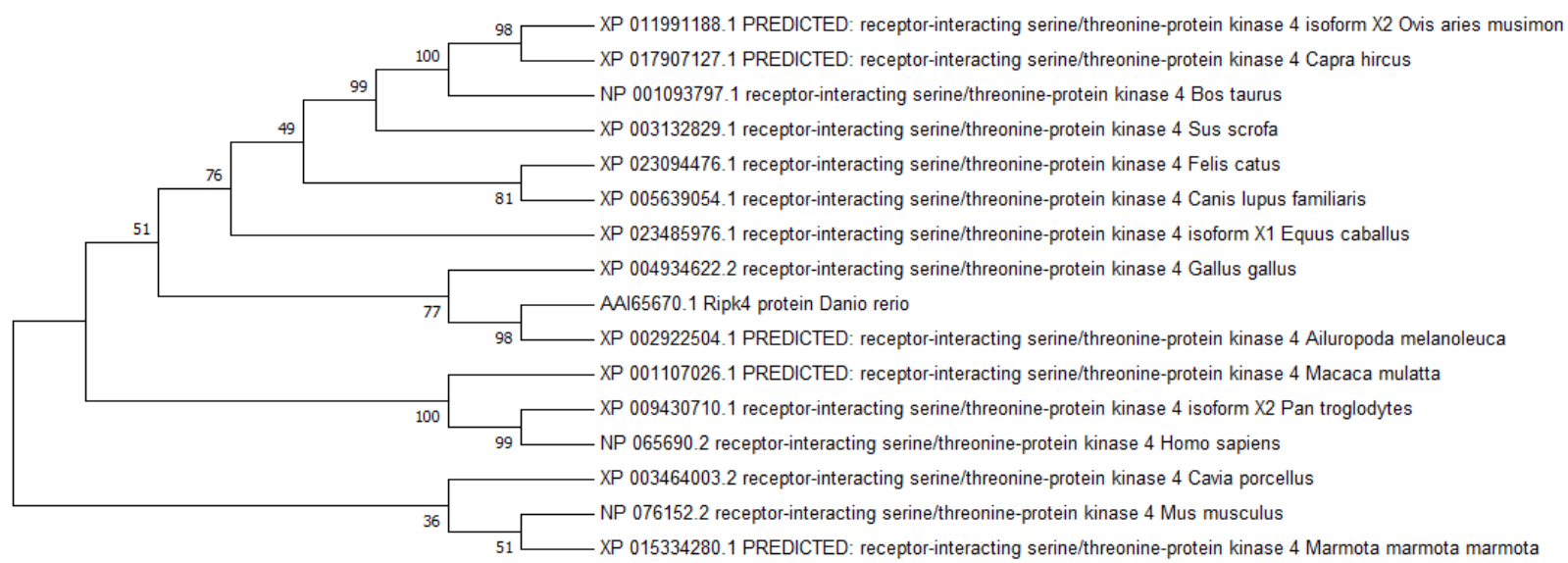

Figure 8. Phylogenetic tree of all 15 orthologs with 1000 bootstrap repetitions

\section{Discussion}

RIPK4 works in Protein Kinase C (PKC) pathways [9]. Protein Kinase $\mathrm{C}$ is a family of proteins that is responsible for controlling the function of other proteins by inhibiting or accelerating their function [35]. RIPK4 gene is responsible for encoding serine/threonineprotein kinase which interacts with kinase Delta-C protein. RIPK 4 protein plays a vital role in the development of epithelial membrane and can be responsible for Popliteal Pterygium Syndrome [5]. To identify its role and functionality, in silico study of protein RIPK4 wild type and its both mutants I121A and T184I were conducted. Secondary and tertiary structure prediction and Evaluation, docking interaction, Pocket identification, and Phylogenetic analysis were performed. 
PSIPRED results indicated the change in the number of residues in helices, strands, and coils of the secondary structures of RIPK4 and both I121A and T184I mutants. Homology modeling of RIPK4 protein and both mutants spotted major differences among their secondary and tertiary structures; even slight modification was verified through superimposition by aligning the RIPK4 protein with its mutants individually. The structures were then evaluated by RAMPAGE where the maximum percentage of residues lying in the favorable region indicated the reliability of predicted structures. CASTp results showed that the total number of active sites identified in RIPK4 and both I121A and T184I mutants were different. The active sites in RIPK4 were 130 while in I121A mutant active sites were minimized to 119 and T184I mutant contained 112 active sites.

The docking results showed diversity not only in the interacting residues of RIPK4 (wild type \& mutants) with ligands and protein but also depicted changes in hydrogen bonds, hydrophilic and hydrophobic interactions.

T-Coffee results revealed $100 \%$ amino acid conservation in all 15 orthologs species that indicates its importance in protein conserved region and protein functionality. Phylogenetic analysis by MEGA7 revealed that human beings are phylogenetically most closely related to Pan troglodytes. It concluded that in addition to sequence similarity between both organisms, functionality is also the same in closely related species and hence any structural change can lead to the disruption of normal function and can lead to the disease. These results revealed the importance of these mutated amino acids and it also revealed that it does not affect the structure of protein only but also its active sites thus leading to an alteration in the protein function resulting in the disease state.

\section{Conclusion}

In this study, the focus relies upon the analysis of wild-type RIPK4 protein and both I121A and T184I mutants, their secondary and tertiary structures, interacting residues, and the sites involved in protein-protein and protein-ligand docking along with their conformational changes provided. The variations in the predicted secondary structure of RIPK4 and both I121A and T184I mutants confirm the effect of the mutation on the structures. The predicted tertiary structures through MODELLER were superimposed to identify the change in the structures due to mutation. The predicted structures were evaluated using the Ramachandran Plot approach of RAMPAGE software to identify the reliability of the predicted structures. CASTp tool was used to identify the active sites of the RIPK4 protein and its mutants. CASTp results showed that active sites in RIPK4 were 130 while in I121A mutant active sites were minimized to 119 and T184I mutant contained 112 active sites. The docking results of results were analyzed using DimPlot of LigPlus to identify the difference among interacting residues. The docking results showed diversity not only in the interacting residues of RIPK4 (wild type \& mutants) with ligands and protein but also depicted changes in hydrogen bonds, hydrophilic and hydrophobic interactions. For the phylogenetic analysis, a total of 15 orthologs were selected and retrieved from the Ensembl genome browser, and a phylogenetic tree was constructed to observe the evolution. The bootstrap method identifies Pan troglodytes as the nearest ancestor of humans. These findings can be utilized in novel drug discovery and development and can ignite advancements in therapeutic development. This in-silico analysis of proteins has paved the paths towards better insights into diseases hence, providing precise, cost-effective, and accurate therapeutic approaches. 


\section{Authors' contributions}

Conceived and designed the experiments: MJU Hasnain \& MT Pervez, Performed the experiments: A Sarwar \& $\mathrm{T}$ Anwar, Analyzed the data: MJU Hasnain, Contributed reagents/ materials/ analysis tools: T Aslam, Wrote the paper: MJU Hasnain, A Sarwar \& MT Pervez.

\section{References}

1. Bahetwar SK, Pandey RK \& Bahetwar TS (2011). Popliteal pterygium syndrome: orofacial and general features. J Indian Soc Pedod Prev Dent 29(4): 333335.

2. Sisti A, Freda N, Giacomina A \& Gatti GL (2017). Popliteal pterygium syndrome with syngnathia. $J$ of Craniofacial Surgery 28(3): e250-e251.

3. Hammond NL, Dixon J \& Dixon MJ (2019). Periderm: Life-cycle and function during orofacial and epidermal development. Semin Cell Dev Biol 91: 75-83.

4. Moriarty NW, Liebschner D, Klei HE, Echols N, Afonine PV, Headd JJ \& Adams PD (2018). Interactive comparison and remediation of collections of macromolecular structures. Protein Sci 27(1): 182-194.

5. Aslan Y, Erduran E \& Kutlu N (2000). Autosomal recessive multiple pterygium syndrome: a new variant. Am J Med Genet 93(3): 194-197.

6. Bähr C, Rohwer A, Stempka L, Rincke G, Marks F \& Gschwendt M (2000). DIK, a novel protein kinase that interacts with protein kinase $\mathrm{C} \delta$ : cloning, characterization, and gene analysis. $J$ of Biol Chem 275(46): 36350-36357.

7. Abdalla EM \& Morsy H (2011). Bartsocas-Papas syndrome: unusual findings in the first reported egyptian family. Case reports in genetics, 2011.

8. Chen L, Haider K, Ponda M, Cariappa A, Rowitch D \& Pillai S (2001). Protein kinase C-associated kinase (PKK), a novel membrane-associated, ankyrin repeat-containing protein kinase. $J$ of Biol Chem 276(24): 21737-21744.

9. Huang $\mathrm{X}$, McGann JC, Liu BY, Hannoush RN, Lill JR, Pham V \& Dixit VM (2013). Phosphorylation of Dishevelled by protein kinase RIPK4 regulates Wnt signaling. Sci 339(6126): 1441-1445.

10. Kalay E, Sezgin O, Chellappa V, Mutlu M, Morsy H, Kayserili H \& Akarsu NA (2012). Mutations in RIPK4 cause the autosomal-recessive form of popliteal pterygium syndrome. The Am J of Human Gene 90(1): 76-85.

11. Huang CS, Oberbeck N, Hsiao YC, Liu $\mathrm{P}$, Johnson AR, Dixit VM \& Hymowitz SG (2018). Crystal structure of RIPK4 reveals dimerization-dependent kinase activity. Structure 26(5): 767-777.

12. Chirieleison SM, Kertesy SB \& Abbott DW, (2016). Synthetic Biology Reveals the Uniqueness of the RIP Kinase Domain. J Immunol 196(10): 4291-4297.

13. Mitchell K, O'Sullivan J, Missero C, Blair E, Richardson R, Anderson B \& Dixon MJ (2012). Exome sequence identifies RIPK4 as the Bartsocas-Papas syndrome locus. The Am J of Human Gene 90(1): 69-75.

14. Gripp KW, Ennis S \& Napoli J (2013). Exome analysis in clinical practice: expanding the phenotype of BartsocasPapas syndrome. Am J Med Genet A 161A(5): 1058-1063.

15. Richardson R, Mitchell K, Hammond NL, Mollo MR, Kouwenhoven EN, Wyatt ND \& Dixon J (2017). p63 exerts Spatio-temporal control of palatal epithelial cell fate to prevent cleft palate. PLoS Gene 13(6): e1006828.

16. Wheeler DL, Barrett T, Benson DA, Bryant SH, Canese K, Chetvernin V \& Khovayko O (2005). Database resources of the National Center for Biotechnology Information. Nucleic Acids Res. 
17. McGuffin LJ, Bryson K \& Jones DT (2000). The PSIPRED protein structure prediction server. Bioinform 16(4): 404405.

18. Webb B, \& Sali A (2016). Comparative protein structure modeling using MODELLER. Current Protocols in Bioinform 54(1): 5-6.

19. Schymkowitz J, Borg J, Stricher F, Nys R, Rousseau F \& Serrano L (2005). The FoldX web server: an online force field. Nuc Acids Res 33(suppl_2): W382W388.

20. Colovos C \& Yeates TO (1993). Verification of protein structures: patterns of nonbonded atomic interactions. Protein Sci 2(9): 1511-1519.

21. Mooers BH (2016). Simplifying and enhancing the use of PyMOL with horizontal scripts. Protein Sci 25(10): 1873-1882.

22. Rigsby RE \& Parker AB (2016). Using the $\mathrm{P}$ y MOL application to reinforce visual understanding of protein structure. Biochem and Mol Biol Edu 44(5): 433437.

23. Binkowski TA, Naghibzadeh S \& Liang J (2003). CASTp: Computed Atlas of Surface Topography of proteins. Nucl Acids Res 31(13): 3352-3355.

24. Bhattacharya D, Nowotny J, Cao R, \& Cheng J (2016). 3Drefine: an interactive web server for efficient protein structure refinement. Nucl Acids Res 44(W1): W406-W409.

25. Macindoe G, Mavridis L, Venkatraman V, Devignes MD \& Ritchie DW (2010). HexServer: an FFT-based protein docking server powered by graphics processors. Nucl Acids Res 38(suppl_2): W445-W449.

26. Dallakyan S \& Olson AJ (2015). Smallmolecule library screening by docking with PyRx. In Chemical biology (pp. 243-250). Humana Press, New York, NY. 27. Wallace AC, Laskowski RA \& Thornton JM (1995). LIGPLOT: a program to generate schematic diagrams of proteinligand interactions. Protein Engineering, Design, and Selection, 8(2): 127-134.

28. Zhou P, Tian F \& Shang Z (2009). 2D depiction of nonbonding interactions for protein complexes. J Comput Chem 30(6): 940-951

29. Carver T, \& Bleasby A (2003). The design of Jemboss: a graphical user interface to EMBOSS. Bioinform 19(14): 1837-1843.

30. Olson SA (2002). EMBOSS opens up sequence analysis. European Molecular Biology Open Software Suite. Brief Bioinform 3(1): 87-91.

31. Poirot O, O'Toole E \& Notredame C (2003). Tcoffee@igs: A web server for computing, evaluating, and combining multiple sequence alignments. Nucl Acids Res 31(13): 3503-3506.

32. Kumar S, Stecher G \& Tamura K (2016). MEGA7: Molecular Evolutionary Genetics Analysis Version 7.0 for Bigger Datasets. Mol Biol Evol 33(7): 18701874.

33. Aiyar A (2000). The use of CLUSTAL W and CLUSTAL $\mathrm{X}$ for multiple sequence alignment. In Bioinformatics methods and protocols (pp. 221-241). Humana Press, Totowa, NJ.

34. Choi JH, Jung HY, Kim HS \& Cho HG (2000). PhyloDraw: a phylogenetic tree drawing system. Bioinform 16(11): 10561058.

35. Kikkawa U, Kishimoto A \& Nishizuka Y (1989). The protein kinase C family: heterogeneity and its implications. Annu Rev Biochem 58: 31-44. 